\title{
Arrested Development: Slow Progression of Fibrosis in Patients with NAFLD and IBD
}

\author{
Yejoo Jeon ${ }^{1} \cdot$ Berkeley N. Limketkai ${ }^{1}$
}

Accepted: 9 October 2020 / Published online: 22 October 2020

(c) Springer Science+Business Media, LLC, part of Springer Nature 2020

Nonalcoholic fatty liver disease (NAFLD) that has recently emerged as one of the most common chronic liver diseases worldwide is a significant contributor to hepatic cirrhosis and hepatocellular carcinoma [1]. Following general population trends, NAFLD has also been increasingly recognized among patients with inflammatory bowel diseases (IBD). A recent meta-analysis estimated NAFLD to be present in approximately $28 \%$ of patients with IBD and more commonly among patients with features of severe IBD, such as longer disease duration or a history of abdominal surgery [2]. A proposed mechanism for this observation suggests that an increased systemic inflammatory burden from IBD contributes to hepatic steatosis; such inflammation could theoretically drive progression of hepatic fibrosis. Nevertheless, the clinical implications of the concurrent occurrence of IBD and NAFLD are yet unclear.

The natural history of NAFLD may vary greatly among patients, with some individuals experiencing a clinically benign course and others progressing to advanced liver disease. This disparity in outcomes and the morbidity of the more severe complications highlight the importance of proper disease diagnosis and prognosis. Prior studies have shown hepatic fibrosis to be the most reliable marker associated with overall and liver-related mortality in patients with NAFLD, although features of inflammation or the NAFLD activity score (correlated with the presence of NASH) were not $[3,4]$. Though fibrosis is usually a histologic diagnosis, with the rising prevalence of NAFLD combined with the dynamic nature of fibrosis, variable disease trajectory, and risks associated with liver biopsy, it would be impractical to monitor NAFLD-associated fibrosis in large populations solely through biopsy [5]. As such, noninvasive radiologic

Berkeley N. Limketkai

berkeley.limketkai@gmail.com

1 Vatche and Tamar Manoukian Division of Digestive Diseases, UCLA School of Medicine, 100 UCLA Medical Plaza Suite \#345, Los Angeles, CA 90095, USA and serum-based tests have emerged as potential alternatives to monitor fibrosis progression in NAFLD. In the context of patients with IBD, many who already undergo numerous invasive procedures, receive potent immunosuppressive medications, and endure recurrent flares, the availability of minimally or noninvasive measures to monitor disease activity becomes increasingly important.

In this issue of Digestive Diseases and Sciences, Ritaccio et al. [6] report their findings on the prevalence of NAFLD and trends in the progression of NAFLD-associated fibrosis among patients with IBD. In a large population of 1672 patients with IBD seen at a tertiary-care academic center, $14 \%$ had radiologic or biopsy-proven evidence of hepatic steatosis. The investigators also employed the NAFLD fibrosis score (NFS) - a validated noninvasive scoring system based on readily available clinical and laboratory measures-to stage hepatic fibrosis. Of 207 IBD patients with NAFLD, an index NFS was obtained from 138 patients. More than half of these patients had fibrosis scores of F0-F2 (no fibrosis to moderate fibrosis), whereas very few patients (4\%) had F3 or F4 (advanced fibrosis to cirrhosis). Nearly a third of the patients $(31 \%)$ fell within the indeterminate range. These findings were consistent with other investigators' observations that most patients with comorbid IBD and NAFLD did not have significant fibrosis (F3 or F4) [7, 8]. The other studies also did not find measures of fibrosis to be associated with IBD-related factors or IBD medications.

Of greater novelty and interest, the current study evaluated longitudinal progression of fibrosis in this population of patients with comorbid IBD and NAFLD. Among 56 patients at 5 years, $77 \%$ had a stable NFS, $16 \%$ had progression of NFS, and $7 \%$ had improvement of NFS. Stratified by exposure at any time to different medications (i.e., biologic therapy, methotrexate, thiopurine), the distribution of NFS changes remained similar. These results suggest that hepatic fibrosis progression among IBD patients with NAFLD is uncommon. 
The coupled findings that most patients with IBD and NAFLD do not have significant fibrosis at baseline and do not experience rapid hepatic disease progression are reassuring. In the current state of evidence where available data on the interaction between IBD and NAFLD are sparse, this study provides valuable insight into the relevant epidemiology and clinical outcomes. There are nonetheless caveats to consider when interpreting the presented data. For one, since the sample size of patients with comorbid IBD and NAFLD is small with the sample size of those with longitudinal follow-up even smaller, additional studies are clearly needed to confirm the findings. Secondly, though the NFS is a validated scoring system, its applicability in patients with IBD and coexisting NAFLD is unclear. Variables used to calculate the NFS are age, body mass index, impaired fasting glucose or diabetes, platelet count, albumin, and aspartate aminotransferase (AST)-to-alanine aminotransferase (ALT) ratio. IBD is a dynamic disease that can lead to acute fluctuations in body mass index, platelet count, and albumin. Even during periods of clinical remission (a frequently used marker of baseline disease activity in studies of NAFLD and IBD), underlying endoscopic and histologic disease activity could still influence these markers, which could consequently affect NFS scoring. Therefore, further studies are needed to validate NFS and other noninvasive markers of hepatic fibrosis in this specific population. Thirdly, although the NFS provides a rapid method for assessing fibrosis severity in patients with NAFLD, it cannot clearly discriminate milder fibrosis stages. Though it incorporates two thresholds to distinguish non-moderate fibrosis from advanced fibrosis, this study noted a third of patients falling in the "indeterminant" range. There is a dose-response gradient of worsening outcomes throughout the spectrum of fibrosis stages. Even at stage F1, all-cause mortality in patients with NAFLD is increased when compared with stage F0. Therefore, the NFS is unable to provide risk stratification in patients with early stages of fibrosis. This limitation could theoretically be addressed by adding another approach in a multi-staged assessment with initial NFS followed by radiologic or other noninvasive modality [9]. Monitoring for disease progression could also be addressed by comparing numerical mean NFS values in addition to only using ordinal classifiers [10].

Recent research has highlighted the notable prevalence of NAFLD among patients with IBD, as well as the potential interactions between these two diseases. Ritaccio and colleagues [6] take the further step to investigate the clinical implications of NAFLD in IBD. They provided important and reassuring data that most patients have low-intermediate fibrosis that largely remains stable over 5 years. As with the early investigation of any disease process, additional studies are needed to build on the foundations of these results and improve our understanding on how to best manage patients with NAFLD and IBD.

Authors' Contribution YJ and BNL prepared, revised, and approved the manuscript.

Funding None.

\section{Compliance with Ethical Standards}

Conflict of interest The authors declare that they have no conflict of interest.

\section{References}

1. Sanyal AJ. Past, present and future perspectives in nonalcoholic fatty liver disease. Nat Rev Gastroenterol Hepatol. 2019;16:377386. https://doi.org/10.1038/s41575-019-0144-8.

2. Zou ZY, Shen B, Fan JG. Systematic review with meta-analysis: epidemiology of nonalcoholic fatty liver disease in patients with inflammatory bowel disease. Inflamm Bowel Dis. 2019;25:17641772. https://doi.org/10.1093/ibd/izz043.

3. Angulo P, Kleiner DE, Dam-Larsen S, et al. Liver fibrosis, but no other histologic features, is associated with long-term outcomes of patients with nonalcoholic fatty liver disease. Gastroenterology. 2015;149:389-97.e10. https://doi.org/10.1053/j.gastr o.2015.04.043.

4. Dulai PS, Singh S, Patel J, et al. Increased risk of mortality by fibrosis stage in nonalcoholic fatty liver disease: systematic review and meta-analysis. Hepatology. 2017;65:1557-1565. https://doi. org/10.1002/hep.29085.

5. Ekstedt M, Nasr P, Kechagias S. Natural history of NAFLD/ NASH. Curr Hepatol Rep. 2017;16:391-397. https://doi. org/10.1007/s11901-017-0378-2.

6. Ritaccio G, Stoleru G, Abutaleb A, et al. Non-alcoholic fatty liver disease is common in IBD patients however progression to hepatic fibrosis by noninvasive markers is rare. Dig Dis Sci. (Epub ahead of print). https://doi.org/10.1007/s10620-020-06588-6.

7. Magrì $\mathrm{S}$, Paduano $\mathrm{D}, \mathrm{Chicco} F$, et al. Nonalcoholic fatty liver disease in patients with inflammatory bowel disease: beyond the natural history. World J Gastroenterol. 2019;25:5676-5686. https ://doi.org/10.3748/wjg.v25.i37.5676.

8. Carr RM, Patel A, Bownik H, et al. Intestinal inflammation does not predict nonalcoholic fatty liver disease severity in inflammatory bowel disease patients. Dig Dis Sci. 2017;62:1354-1361. https://doi.org/10.1007/s10620-017-4495-0.

9. Agbim U, Asrani SK. Non-invasive assessment of liver fibrosis and prognosis: an update on serum and elastography markers. Expert Rev Gastroenterol Hepatol. 2019;13:361-374.

10. Salomone F, Micek A, Godos J. Simple scores of fibrosis and mortality in patients with NAFLD: a systematic review with metaanalysis. J Clin Med. 2018;7.

Publisher's Note Springer Nature remains neutral with regard to jurisdictional claims in published maps and institutional affiliations. 